\title{
Validação do Método da Reflectância no Infravermelho Proximal para Análise de Silagem de Milho 1
}

\section{Roberto Serena Fontaneli ${ }^{2}$, João Walter Durr ${ }^{3}$, Simone Meredith Scheffer-Basso ${ }^{4}$, Fabrício Haubert ${ }^{5}$, Fernanda Bortolini ${ }^{6}$}

\begin{abstract}
RESUMO - Os métodos tradicionais de análise da qualidade nutricional de forragens são demorados e de custo elevado. O método da reflectância no infravermelho proximal apresenta-se uma alternativa que não utiliza reagentes, não é destrutivo e é extremamente rápido. No entanto, para que seja utilizado rotineiramente, faz-se necessário o desenvolvimento de curvas de calibração, obtidas por intermédio de equações matemáticas a partir dos resultados de ensaios laboratoriais. O presente trabalho foi desenvolvido com o objetivo de desenvolver curvas de calibração para silagem de milho, utilizando-se um total de 246 amostras, oriundas de diferentes regiões do Rio Grande do Sul. O material foi analisado quanto aos teores de proteína bruta (PB), matéria seca (MS), fibra em detergente ácido (FDA), fibra em detergente neutro (FDN) e minerais (Ca, K, Mg, P) pelos métodos analíticos de referência. As mesmas amostras foram analisadas em um espectrômetro, cuja leitura foi realizada nos comprimentos de onda de 1100 a 2500 nanômetros. Para a calibração, foi utilizado o tratamento matemático do programa Match e, posteriormente, análise de regressão, tendo como variáveis independentes os resultados das análises físico-químicas e, como variáveis dependentes, os resultados da reflectância no infravermelho proximal. A avaliação do grau de ajuste foi feita por intermédio dos coeficientes de determinação ( $\mathrm{R}^{2}$ ). A acurácia foi elevada $\left(\mathrm{R}^{2}=0,99\right)$ para $\mathrm{PB}, \mathrm{FDA}, \mathrm{FDN}$ e MS, indicando a validade do método na predição desses parâmetros em silagem de milho. Mesmo que o $\mathrm{R}^{2}$ para determinação de minerais tenha variado de 0,92 a 0,94 , ainda são necessários estudos detalhados do comportamento do espectro, para avaliar o tipo de complexo mineral formado e sua relação com o método físicoquímico. Os resultados médios de PB, FDN, FDA e MS foram de 7,86; 60,66; 29,87 e 94,17\%, respectivamente. Os resultados médios para $\mathrm{Ca}, \mathrm{P}, \mathrm{K}$ e $\mathrm{Mg}$ foram de 0,$27 ; 0,21 ; 1,14$ e $0,22 \%$, respectivamente.
\end{abstract}

Palavras-chave: métodos de análise de forragem, proteína, fibra, matéria seca, minerais

\section{Validation of the Near Infrared Reflectance Method for the Analysis of Corn Silage}

ABSTRACT - Traditional analytical methods of assessing forage nutritional value are time-consuming and expensive. The near infrared reflectance method appears as an alternative that does not utilize chemicals, is not destructive and is extremely fast. However, in order to be used in a routinely basis, calibration curves have to be developed from laboratory results and mathematical equations. The objective of this paper was to develop calibration curves for corn silage, using a total of 246 samples collected in different regions of Rio Grande do Sul. The samples were analyzed using the reference methods to determine crude protein (CP), dry matter (DM), acid detergent fiber (ADF), neutral detergent fiber (NDF) and minerals ( $\mathrm{Ca}, \mathrm{K}, \mathrm{Mg}$ and $\mathrm{P}$ ). The same samples were also analyzed by spectroscopy, using a wave length region that ranges from 1100 to $2500 \mathrm{~nm}$. Data was mathematically treated by the Match program and then a regression model was fitted, having the results from the chemical analysis as independent variables and the results from the near infrared reflectance as dependent variables. The evaluation of the adjustment level was made through the determination coefficients $\left(\mathrm{R}^{2}\right)$. The accuracy was elevated $\left(\mathrm{R}^{2}=0.99\right)$ for $\mathrm{CP}, \mathrm{ADF}, \mathrm{NDF}$ and $\mathrm{DM}$, indicating the validity of this method to predict such parameters in corn silage. Even though the $\mathrm{R}^{2}$ for minerals have ranged from 0.92 to 0.94 , further research about the behavior of the spectrum is needed in order to evaluate the type of mineral complex formed and its relation with the chemical method. The averages for CP, NDF, ADF and DM were 7.86, 60.66, 29.87 and $94.17 \%$, respectively. The averages for $\mathrm{Ca}, \mathrm{P}, \mathrm{K}$ and $\mathrm{Mg}$ were, respectively, 0.27 , $0.21,1.14$ and $0.22 \%$.

Key Words: forage analysis methods, protein, fiber, dry matter, minerals.

\footnotetext{
1 Trabalho realizado com apoio financeiro da Fundação de Amparo à Pesquisa do Rio Grande do Sul (Fapergs).

${ }^{2}$ Eng.-Agr., MSc., Professor da Universidade de Passo Fundo (UPF). Caixa Postal 611, Passo Fundo, Cep: $99001-970$.

E.mail: roberto@upf.tche.br

3 Eng.-Agr., PhD., Professor da UPF. E.mail: durr@upf.tche.br

${ }^{4}$ Eng.-Agr., Dr.,Professor da UPF. E.mail: simone@upf.tche.br

${ }^{5}$ Acadêmico do curso de Medicina Veterinária da UPF e bolsista do CNPq.

${ }^{6}$ Acadêmica do curso de Biologia da UPF e bolsista da Fapergs.
} 


\section{Introdução}

No Rio Grande do Sul, especialmente nas regiões do Planalto Médio e Missões, a atividade leiteira tem sido incrementada nos últimos anos, em pequenas e médias propriedades. O sistema de forrageamento dos animais é à base de pastagens cultivadas, mas a silagem de milho (Zea mays L.) é imprescindível, pois os meses do outono e inverno apresentam marcada diminuição na oferta de pasto. Nesse sentido, as cooperativas do setor têm incentivado os produtores a enviarem amostras das forragens disponíveis na propriedade para análise do valor nutritivo, com objetivo de racionalizar a suplementação com rações, reduzindo custos.

Os parâmetros de valor nutritivo mais importantes para tal finalidade são proteína bruta (PB), fibra em detergente ácido (FDA), fibra em detergente neutro (FDN) e energia líquida de lactação. O método tradicional de análise bromatológica tem sido amplamente utilizado nos laboratórios nacionais; no entanto, é um método demorado e de custo elevado. Como método alternativo, tem-se a espectrometria de reflectância no infravermelho proximal (NIRS). Trata-se de um método potencialmente preciso, não destrutivo e com predição de características nutricionalmente relevantes, inclusive de silagens (Alomar et al., 1999). Sua grande vantagem em relação ao método tradicional está na análise múltipla dos constituintes, menor necessidade de mão-deobra, rapidez e, portanto, menor custo, além de não ser poluente por não utilizar reagentes.

O princípio de análise NIRS consiste na absorção da luz infravermelha próximal (1100 a $2500 \mathrm{~nm}$ ) por compostos orgânicos. O método se baseia no fato de que cada um dos principais componentes das forragens tem características de absorção específicas, onde há vibrações das ligações hidrogenadas induzidas pelo calor nos grupos funcionais das moléculas (Marten et al., 1985). Assim, pelo NIRS há condições de prognosticar o conteúdo dos diferentes componentes nutricionais, através de equações de calibração para cada um desses (Kjos, 1990b). Segundo Kjos (1990a), na ausência de boas rotinas de análise de forragens, o NIRS pode ser uma alternativa eficaz se as curvas de calibração forem muito bem realizadas. O autor obteve boas correlações entre a digestibilidade in vitro de matéria orgânica e a FDN e FDA prognosticadas pelo NIRS, em silagem de milho.
Ao se desenvolver uma calibração, relacionamse às informações espectrais com as informações de referência (composição química), definindo o tratamento matemático dos dados, o segmento do espectro a incluir e o método de regressão a empregar. No desenvolvimento de equações, uma das primeiras fases, a atender, é detectar amostras aberrantes (outliers), que não se encaixam ou não correspondem à calibração. Em silagens, um dos coeficientes mais elevados é para PB e degradabilidade ruminal potencial $\left(\mathrm{R}^{2}=0,99\right)$, enquanto que, para digestibilidade in vivo, é menor $\left(\mathrm{R}^{2}=0,82\right)$ (Alomar \& Fuchlocher, 1998).

Este trabalho teve como objetivo principal o desenvolvimento de curvas de calibração para silagem de milho, com a finalidade de utilizar o NIRS rotineiramente nas predições do valor nutritivo dessa forragem, promovendo um rápido retorno dos resultados aos produtores de leite.

\section{Material e Métodos}

Foram analisadas, no Centro de Pesquisa em Alimentação (Cepa) da Univesidade de Passo Fundo, 246 amostras de silagem de milho, oriundas de diferentes regiões do Rio Grande do Sul, recebidas entre junho de 1996 a dezembro de 1999. As amostras antes de serem analisadas foram secadas a uma temperatura de $60^{\circ} \mathrm{C}$ sob circulação de ar por 48 horas. Após a secagem, as amostras foram moídas $(1 \mathrm{~mm})$ em moinho tipo Wiley, acondicionadas em vidros e submetidas, primeiramente à leitura no NIRS para o armazenamento do espectro das mesmas. Posteriormente, as amostras foram analisadas pelo método físico-químico para os seguintes parâmetros: matéria seca (MS), através de secagem a $105^{\circ} \mathrm{C}$ por 24 horas, PB, pelo método Kjedhal (AOAC, 1995), FDN e FDA, segundo Goering \& Van Soest (1970) e minerais $(\mathrm{Ca}, \mathrm{P}, \mathrm{K}, \mathrm{Mg})$, por absorção atômica. Todas as determinações foram feitas em duplicatas. Pelo método NIRS, as análises foram realizadas em um espectrômetro Perstorp analytical, Silver Spring, MD, modelo 5000, acoplado a um microcomputador equipado com software ISI versão 4.1 (Infrasoft International, University, Park, PA). A leitura foi realizada utilizando-se os comprimentos de onda de 1100 a 2500 nanômetros . Para a calibração, os resultados de análise foram selecionados pelo programa Select e Center, visando escolher as amostras representativas da população-alvo, com base na dis- 
tância Mahalanobis $(\mathrm{H}=0,6)$ entre a amostra e seu grupo e, desse com sua vizinhança (Shenk \& Westerhaus, 1991). Para a geração das equações, foi utilizado o tratamento matemático do programa Match (Shenk \& Westerhaus, 1993). Posteriormente, fez-se análise de regressão, tendo como variável independente os resultados das análises físico-químicas e, como variável dependente, os resultados do NIRS. A avaliação do grau de ajuste foi realizada por intermédio dos coeficientes de determinação obtidos $\left(\mathrm{R}^{2}\right)$.

\section{Resultados e Discussão}

Das 246 amostras analisadas, foram eliminadas menos de $10 \%$ de outliers, indicando que a população-alvo foi muito bem representada, ficando dentro das recomendações (Tabela 1), de acordo com o manual de operação de rotina e calibração do NIRS (Newisi-NIRS2, 1996). Com relação ao grau de ajuste das equações, os valores de $\mathrm{R}^{2}$ e erros-padrões indicam uma elevada acurácia do método NIRS para os parâmetros analisados em silagem de milho. Segundo Shenk \& Westerhaus (1993), isso normalmente se obtém quando se trabalha com uma única espécie. Destacam-se os baixos erros-padrões de FDN, FDA e MS, basicamente devido à melhor precisão das técnicas de referência (Shenk \& Westerhaus, 1993). No caso de MS isso se deve, principalmente, à pronta determinação após a leitura do espectro, indicando a importância dos procedimentos de análise. Com alfafa (Medicago sativa L.), Pires \& Prates (1998), não obtiveram boa correlação para MS, atribuído a problemas ocorridos no armazenamento das amostras.

Para proteína bruta (Tabela 2), os valores obtidos ficaram numa amplitude de 5,43 a 10,52\%, com média de 7,86\%, condizendo com a literatura (Almeida Filho et al.,1999; Lavezzo et al., 1997; Neiva et al., 1998); sendo a silagem de milho um material, tipicamente, com baixos teores de proteína. A elevada amplitude, porém, pode ser atribuída às diferentes proporções de caule, folha e espiga nas amostras, que eram, pela sua própria origem, muito heterogêneas. Assim, amostras compostas por maiores proporções de folhas e espigas, normalmente, apresentam teores mais elevados de proteína, uma vez que os caules possuem menor conteúdo desse componente, conforme verificado por Lavezzo et al. (1997). O alto coeficiente de determinação obtido $\left(\mathrm{R}^{2}=0,99\right)$ demonstra a validade do método para tal parâmetro. Destaca-se, porém, que os valores de PB obtidos, tanto pelo método NIRS, quanto pelo tradicional, podem estar subestimados, pela volatilização do $\mathrm{N}$ amoniacal (Wernli \& Ojeda, 1990).

O teor de FDN $(60,66 \%)$ foi elevado, se comparado com resultados de Almeida Filho et al. (1999) e

Tabela 1 - Número de amostras ( $\left.{ }^{\circ}\right)$, desvio-padrão (DP), coeficiente de determinação ( $\mathrm{R}^{2}$ ), erro-padrão da validação cruzada $(E P C)$, erro-padrão da validação (EPV), coeficiente de determinação da validação $\left(R^{2} \vee\right)$ e tratamento matemático (TM) das análises para os parâmetros analisados em silagem de milho

Table 1 - Number of samples (N), standard deviation (SD), coefficient of determination $\left(\mathrm{R}^{2}\right)$, crossed validation standard error (CSE), validation standard error (VSE), validation determination coefficient $\left(R^{2} v\right)$ and mathematical treatment (MT) used for the analyzed parameters in corn silage

\begin{tabular}{|c|c|c|c|c|c|c|c|}
\hline $\begin{array}{l}\text { Parâmetro } \\
\text { Parameter }\end{array}$ & $\begin{array}{l}\mathrm{N}^{\circ} \\
N\end{array}$ & $\begin{array}{l}\text { DP } \\
S D\end{array}$ & $\begin{array}{l}\mathrm{R}^{2} \\
R^{2}\end{array}$ & $\begin{array}{l}\text { EPC } \\
C S E\end{array}$ & $\begin{array}{l}\text { EPV } \\
V S E\end{array}$ & $\begin{array}{l}\mathrm{R}^{2} \mathrm{~V} \\
R_{V}^{2}\end{array}$ & $\begin{array}{l}\text { TM } \\
M T\end{array}$ \\
\hline $\mathrm{PB}$ & 220 & 1,08 & 0,98 & 0,14 & 0,16 & 0,98 & $1-4-4-1$ \\
\hline $\begin{array}{l}C P \\
\text { FDN } \\
N D F\end{array}$ & 229 & 5,14 & 0,99 & 0,44 & 0,51 & 0,99 & $1-4-4-1$ \\
\hline $\begin{array}{l}N D F \\
\text { FDA } \\
A D F\end{array}$ & 221 & 3,84 & 0,99 & 0,32 & 0,39 & 0,99 & $1-4-4-1$ \\
\hline $\begin{array}{l}\text { MS } \\
D M\end{array}$ & 221 & 2,91 & 0,99 & 0,22 & 0,27 & 0,99 & $3-10-10-1$ \\
\hline $\begin{array}{l}\mathrm{Ca} \\
\mathrm{Ca}\end{array}$ & 225 & 0,11 & 0,93 & 0,03 & 0,03 & 0,96 & $1-4-4-1$ \\
\hline $\begin{array}{l}\mathrm{P} \\
P\end{array}$ & 227 & 0,03 & 0,92 & 0,01 & 0,01 & 0,87 & $1-4-4-1$ \\
\hline $\begin{array}{l}\mathrm{K} \\
K\end{array}$ & 223 & 0,25 & 0,94 & 0,08 & 0,06 & 0,89 & $1-4-4-1$ \\
\hline $\begin{array}{l}\mathrm{Mg} \\
M g\end{array}$ & 228 & 0,03 & 0,92 & 0,01 & 0,01 & 0,87 & $1-4-4-1$ \\
\hline
\end{tabular}


Neiva et al. (1998), que obtiveram no máximo, 52\% e $55 \%$, respectivamente. Observa-se, porém, que a elevada amplitude obtida no presente estudo $(49,41$ a $72,35 \%)$, comprova a heterogeneidade das amostras. As silagens analisadas provêm de diferentes híbridos de milho, com prováveis estádios de desenvolvimento, além de distintos processos de ensilagem, enquanto que nos trabalhos citados, as amostras representam ensaios específicos, de um único local, com maior controle de tais variáveis, e, portanto, mais homogêneas. Além disso, há que se considerar também outros fatores que podem interferir nos resultados desse parâmetro. No presente trabalho, a FDN foi analisada segundo Goering \& Van Soest (1970), que não recomendam o uso de enzimas para digestão do amido, o que pode ter provocado uma superestimação desse componente. No entanto, é importante destacar o elevado $\mathrm{R}^{2}=0,99$, considerando-se os resultados de Marten et al. (1983), Pires \& Prates, (1998), entre outros. Já, com relação à FDA, a média obtida (29,87\%) foi similar aos resultados de Almeida Filho et al. (1999), Lavezzo et al. (1997) e Neiva et al. (1998), para silagens de milho. O $\mathrm{R}^{2}$ $(0,99)$ para tal componente confirma os dados de Valdes et. al. (1990).
Para o conteúdo de MS, destaca-se a pequena variação entre as amostras 86,13 e 99,67\%. Ressalta-se que tais valores não indicam os teores de MS da silagem como oferecida, mas sim, a MS residual após secagem a $60^{\circ} \mathrm{C}$, o que explica a pequena amplitude observada. A MS é válida para converter os demais componentes analisados para a base seca. Assim, na sua determinação são especialmente importantes os cuidados com a ambiência no laboratório, o acondicionamento e armazenamento das amostras, para evitar a influência da umidade. Já, o elevado $\mathrm{R}^{2}(0,99)$ foi obtido principalmente, devido a imediata leitura do espectro após a secagem. Com alfafa (Medicago sativa L.), Pires \& Prates (1998) não obtiveram boa correlação para $\mathrm{MS}, \mathrm{R}^{2}=0,93$, atribuído, em parte, às condições ambientais do laboratório.

Com os minerais, conforme esperado (NewisiNIRS2, 1996), obtiveram-se os menores $\mathrm{R}^{2}$. Ocorre que tal fração não sofre alteração pela ação da luz infravermelha (Tabela 2) e, portanto, não existem bandas de absorção para os minerais puros. Segundo Clark et al. (1987), são necessários mais estudos para determinar a relação entre a composição, relativamente elevada, de ácidos orgânicos e a concentração individual de minerais em forragens. $\mathrm{O}$ fato é que os minerais podem estar, tanto complexados organicamente ou sob forma de quelatos, como na forma de sais e íons. Assim, os primeiros poderiam ser determinados, mas as formas iônica e salina não absorvem energia na região do infravermelho proximal.

\section{Conclusões}

Tabela 2 - Valores médios e amplitudes para as características nutricionais analisadas em silagem de milho

Table 2 - Average and range values for the nutritional characteristics of corn silage

\begin{tabular}{lcc}
\hline $\begin{array}{l}\text { Característica } \\
\text { Characteristic }\end{array}$ & $\begin{array}{c}\text { Média (\%) } \\
\text { Average (\%) }\end{array}$ & $\begin{array}{c}\text { Amplitude (\%) } \\
\text { Range (\%) }\end{array}$ \\
\hline PB & 7,87 & $5,43-10,52$ \\
$C P$ & 60,66 & $49,41-72,35$ \\
FDN & & $19,43-40,41$ \\
NDF & 29,87 & \\
FDA & & $86,13-99,67$ \\
$A D F$ & 94,17 & $0,02-0,67$ \\
MS & & $0,10-0,26$ \\
$D M$ & 0,27 & $0,61-2,14$ \\
Ca & 0,21 & $0,12-0,32$ \\
P & 1,14 & \\
K & 0,22 & \\
Mg & &
\end{tabular}

O método NIRS pode ser utilizado na predição de proteína bruta, fibra em detergente ácido, fibra em detergente neutro e matéria seca de silagem de milho seca, com elevada acurácia.

Para minerais são necessários estudos detalhados do comportamento do espectro para avaliar o tipo de complexo mineral formado, sua relação com o método físico-químico e as possíveis interrelações com outros componentes químicos presentes nas forragens.

$\mathrm{Na}$ apreciação dos resultados das análises realizadas pelo NIRS, é importante que sejam considerados fatores como o detalhamento dos métodos analíticos de referência utilizados, estádio de desenvolvimento das plantas, processo de conservação de forragens, obtenção e processamento das amostras. 


\section{Literatura Citada}

ALMEIDA FILHO, S.L.; FONSECA, D.M.; GARCIA, R. Características agronômicas de cultivares de milho (Zea mays L.) e qualidade dos componentes e da silagem. Revista Brasileira de Zootecnia, v.28, n.1, p.7-13, 1999.

ALOMAR, D.; FUCHSLOCHER, R. Fundamentos de la espectroscopia de reflectancia en el infrarojo cercano (NIRS) como metodo de analisis de forrajes. Agro Sur, v.26, n.1, p.88-104, 1998.

ALOMAR, D.; MONTERO, R.; FUCHSLOCHER, R. Effect of freezing and grinding method on near-infrared reflectance (NIR) spectra variation and chemical composition of fresh silage. Animal Feed Science and Technology, v.78, p.5763, 1999.

ASSOCIATION OF OFFICIAL ANALYTICAL CHEMISTS AOAC. Official methods of analysis. 16.ed. Washington: 1995. 2000p.

CLARK, D.H.; MARYLAND, H.F.; LAMB, R.C. Mineral analysis of forages with near infrared reflectance spectrocospy. Agronomy Journal, v.79, n.485-490, 1987.

GOERING, H.K.; Van SOEST, P.J. Forage fiber analysis: apparatus reagents, procedures and some applications. Washington: USDA. 1970. (Agriculture Handb., 379).

KJOS, N.P. Evaluation of the feeding value of fresh forages, silage and hay using near infrared reflectance analysis (NIR). I - A comparison of different methods for predicting the nutritive value. Norwegian Journal Agricultural Science, v.4, p.305-320, 1990a.

KJOS, N.P. Evaluation of the feeding value of fresh forages, silage and hay using near infrared reflectance analysis (NIR). II Effect of drying procedure, type of mill and particle size. Norwegian Journal of Agriculture Science, v.4, p.321330, 1990b.

LAVEZZO, W.; LAVEZZO, O.E.N.M.; CAMPOS NETO, O. Estádios de desenvolvimento do milho. 1. Efeito sobre a produção, composição da planta e qualidade da silagem. Revista Brasileira de Zootecnia, v.26, n.4, p.675-682, 1997.

MARTEN, G.C.; HALGERSON, J.L.; CHERNEY, J.H. Quality prediction of small grain forages by near infrared reflectance spectrocopy. Crop Science, v.23, p.94-96, 1983.

MARTEN, G.C.; SHENK, J.S.; BARTON, F.E. Near infrared reflectance spectroscopy (NIRS). Washington: USDA, 1985. (Agriculture Handb., 643). 96p.
NEIVA, J.N.M.; GARCIA, R.; VALADARES FILHO, S.C. et al. Consumo e digestibilidade aparente de matéria seca e nutriente em dietas à base de silagens e rolão de milho amonizados. Revista Brasileira de Zootecnia, v.27, n.3, p.453-460, 1998.

NEWISI-NIRS2. Routine operation, calibration and network system management software for near infrared instruments. NIRSYSTEMS Inc. Silver Spring, 1996. 368p.

PIRES e PRATES. Uso da técnica da espectrometria de reflectância no infravermelho próximo (NIRS) na predição da composição química da alfafa (Medicago sativa L.). Revista Brasileira de Zootecnia, v.27, n.6, p.1076-1081, 1998.

SHENK, J.; WESTERHAUS, M. New standardization and calibration procedure for NIR analytical systems. Crop Science, v.31, p.1694-1692, 1991.

SHENK, J.; WESTERHAUS, M. Analysis of agriculture and food products by near infrared reflectance spectroscopy. Port Matilda: Penn State University and Infrasoft International, 1993. 116p.

VALDES, E.V.; JONES, G.E.; HOELSTRA, H.J. Effect of growing year and application of a multi-year calibration for predicting quality parameters by near infrared reflectance spectroscopy in whole-plant corn forage. Canadian Journal Plant Science, v.70, p.747-755, 1990.

WERNLI, C.; OJEDA, F. Metodologias para investigaciones sobre conservación y utilización de ensilajes. In: RUIZ, M.E.; RUIZ, A. (Eds.) Nutrición de ruminantes: guia metodológica de investigación. San José - Costa Rica: Instituto Interamericano de Cooperación para la Agricultura: Red de Investigación en Sistemas de Produción Animal en Latinoamérica, 1990. p.179-232. 